\title{
Clinical Characteristics of Diabetic Retinopathy Patients in Eye Clinic of Zainoel Abidin General Hospital Banda Aceh, Indonesia
}

\author{
Lia Meuthia Zaini'), Sri Marlinda'), Annisa Meivira Budiman²) \\ 1)Department of Ophthalmology, Faculty of Medicine, Universitas Syiah Kuala/ \\ Dr. Zainoel Abidin Hospital, Banda Aceh, Indonesia \\ 2)Faculty of Medicine, Universitas Padjadjaran, Bandung, Indonesia
}

\section{ABSTRACT}

Background: Diabetic retinopathy (DR) is one of the most frequent microvascular complications of diabetes mellitus (DM). The increasing number of diabetic retinopathy cases, amajor cause of blindness worldwide, is resulted from the increasing prevalence and life span of diabetes mellitus patients. In 2010, the number of diabetic retinopathy cases was 126.6 million and is predicted to increase to 191.0 million by 2030. The number of patients with vision-threatening diabeticretinopathy is also predicted to increase from 37.3 million to 56.3 million.

Subjects and Method: This study was a descriptive study conducted from January to February 2020.A sample of 98 patients was taken through fixed disease sampling. Research data was collected from electonic medical record of patients visiting Eye Clinic of Zainoel Abidin General Hospital Banda Aceh using total sampling method. The researchers used the triangulation method to complete some missing demographic data in the medical records, such as occupation, education, and monthly salary. Interviews were conducted directly with patients after they underwent an examination, and the results were included in the research form.

Cite this as:

Zaini LM, Marlinda S, Budiman AM (2020). Clinical Characteristics of Diabetic Retinopathy Patients in Eye Clinic of Zainoel Abidin General Hospital Banda Aceh, Indonesia. J Epidemiol Public Health. 05(04): 499507. https://doi.org/10.26911/jepublichealth.2020.05.04.12.

cc) (1) (-) Journal of Epidemiology and Public Health is licensed under a Creative Commons Attribution-NonCommercial-ShareAlike 4.0 International License.

\section{BACKGROUND}

Diabetic retinopathy is the most common microvascular complication of diabetes mellitus (Nentwich et al., 2015). There are two types of diabetic retinopathy: Non
Results: 35 patients (35.7\%) were aged 56-65 years and 26 patients (26.5\%) worked as housewives. The most common systemic complications were heart disorder found in 17 patients (17.3\%). Proliferative diabetic retinopathy (PDR) was identified in 49 patients (50.0\%), whereas 51 patients $(52.0 \%)$ presented with diabetic macular edema (DME). 44 patients (44.9\%) came with less than $6 / 60$ visual acuity on the left eye.

Conclusion: Most patients presenting in Eye Clinic of Zainoel Abidin Hospital Banda Aceh were in worse condition (PDR) with visual acuity of less than 6/60. Therefore, educating and counseling diabetic patients to undergo routine screening by ophthalmologists are important in order to prevent loss of vision caused by diabetic retinopathy.

Keywords: clinical characteristic, diabetic retinopathy

\section{Correspondence:}

Lia Meuthia Zaini. Department of Ophthalmology, Faculty of Medicine, Universitas Syiah Kuala/ Dr. Zainoel Abidin hospital. Jl. Tgk. Daud Deureueh 108, Banda Aceh, Indonesia. Email: lia_mzaini@unsyiah.ac.id. Mobile: +62822-6689-1502. 
venous disorder, whereas PDR is marked by the presence of neovascularization (Duhet al., 2017). Diabetic macular edema is the main cause of blindness in patients with diabetic retinopathy, either early-stage NPDR or late-stage PDR (Ding et al., 2012).

In 2010, more than one-third of 285 million diabetes mellitus patients worldwide exhibit signs of diabetic retinopathy, and another one-third experience severe NPDR and DME. 126.6 million diabetic retinopathy patients in 2010 were predicted to increase to 191.0 million by 2030. The number of patients with vision-threatening diabetic retinopathy (VTDR) was also increased from 37.3 million to 56.3 million(Zheng et al., 2012). The risk factor of diabetic retinopathy can be classified into modifiable and unmodifiable risk factors. Similar to diabetes mellitus, modifiable risk factors of diabetic retinopathy are hypoglycemia, hypertension, dyslipidemia, and obesity. Unmodifiable risk factors of diabetic retinopathy includes age, sex, disease duration of diabetes mellitus, pregnancy, and puberty (Lee et al., 2015).

Damage in retinal microvascularization is manifested as diabetic retinopathy. It also serves as a biomarker of glucose metabolism disorder in systemic microvascularization (Cheung et al., 2008). Both NPDR and PDR are frequently related to other systemic diseases, such as stroke, coronary heart disease, heart failure, and nephropathy (Wat et al., 2016).Other ocular complications are presented as a major cause of morbidity in patients with diabetes mellitus. The most common ocular complications are namely glaucoma, cataract, and dry-eye syndrome. Appropriate control of blood glucose, blood pressure, and lipid levels is important to prevent more severe systemic or ocular complications. Moreover, routine ophthalmology screening is essential for early detection of retinopathy and immediate intervention can be performed (Tajunisah et al., 2011; Khandekar et al., 2010; Sayin et al., 2015).

Physical, social, and economic implications of diabetic retinopathy greatly affect the quality of life of patients. Therefore, patients' awareness and knowledge regarding their disease are essential and related to educational and socioeconomic background. A study about demographic, clinical characteristics, and risk factors of diabetic retinopathy patients in Aceh province has never been conducted. On the other hand, the risk of visual loss caused by diabetic retinopathy can be reduced by effective control of blood glucose and blood pressure, as well as early detection and management of the disease. As a result, a study about demographic and clinical charactertistics of diabetic retinopathy patients isneeded as important information to help health care providers in preventing further damage to the patients. This study aims to determine the clinical characteristics of patients with diabetic retinopathy in the eye clinic of the Zainoel Abidin Hospital.

\section{SUBJECTS AND METHOD}

\section{Study Design}

This was a descriptive study with retrospective design, conducted from January to February 2020 at Eye Clinic of Zainoel Abidin General Hospital Banda Aceh.

\section{Population and Sample}

The population target in this study was all diabetes mellitus patients diagnosed with diabetic retinopathy by ophthalmologists in Eye Clinic of Zainoel Abidin Hospital Banda Aceh. Sample was selected using total sampling. The inclusion criteria were as follow: (1) diabetes mellitus patients admitted to Eye Clinic of Zainoel Abidin Hospital Banda Aceh, (2) had been diagnosed with diabetic 
retinopathy, (3) agree to be a respondent, and (4) able to communicate well. Patients who refused to cooperate would be excluded from the study.

\section{Data Analysis}

After all data were obtained, they will be presented in a table and grouped based on demographic characteristics, clinical characteristics, diabetes characteristics and classification, as well as ocular findings.

\section{Study Instruments}

We did not perform any examinations on the patients because all the data was obtained from the medical record. There were no measurement tools involved.

\section{Research Ethics}

The ethical eligibility in this study is approved by Medical Research Ethical

Table 1. The Demographic Characteristics of Diabetic Retinopathy Patients in Eye Clinic of Zainoel Abidin Hospital Banda Aceh (categorical data)

\begin{tabular}{lcc}
\hline Characteristics & Frequency $(\mathbf{n = 9 8 )}$ & Percentage (\%) \\
\hline Age & 16 & \\
$<45$ years old & 34 & 16.3 \\
$46-55$ years old & 35 & 34.7 \\
$56-65$ years old & 13 & 35.7 \\
$>65$ years old & & 13.3 \\
Sex & 49 & \\
Male & 49 & 50.0 \\
Female & & 50.0 \\
Occupation & 26 & \\
Housewife & 17 & 26.5 \\
Civil servant & 23 & 17.3 \\
Retiree & 6 & 23.5 \\
Private employee & 8 & 6.1 \\
Entrepreneur & 3 & 8.2 \\
Farmer & 15 & 3.1 \\
Unemployed & & $15 \cdot 3$ \\
Education & 42 & \\
College graduates & 21 & 42.9 \\
Senior high school & 10 & 21.4 \\
Junior high school & 10 & 10.2 \\
Elementary school & 15 & 10.3 \\
Not graduated & & 15.3 \\
Monthly Salary & 43 & \\
$<1$ million IDR & 54 & 43.9 \\
1-5 million IDR & 2 & 55.1 \\
5-10 million IDR & 0 & 2.0 \\
$>10$ million IDR & & 0 \\
\hline
\end{tabular}

Committee of Medical Faculty, Universitas Syiah Kuala/ Zainoel Abidin Hospital, Aceh with number: 065/EE/FK-RSUDZA/2020.

\section{RESULTS}

1. Sample Characteristics

Throughout January and February 2020, 98 patients were admitted to Eye Clinic of Zainoel Abidin Hospital Banda Aceh. There were several missing demographic data in the medical records, including occupation, education, and monthly salary. Interviews were then carried out directly to complete this data after they underwent an examination. The resulting demographic data, obtained from both medical records and interviews, are presented in Table 1. 


\section{Data Analysis}

More than $50 \%$ of patients had a history of hypertension and dyslipidemia. Some also had a smoking history. Heart abnormality

Table 2. Risk Factor and Systemic Complications of Diabetic Retinopathy Patients in Eye Clinic of Zainoel Abidin Hospital Banda Aceh (categorical data)

\begin{tabular}{llcc}
\hline \multicolumn{1}{c}{ Characteristics } & \multicolumn{1}{c}{ Category } & Frequency (n=98) & Percentage (\%) \\
\hline Risk Factor & Hypertension & 56 & 57.1 \\
Systemic & Hypercholesterolemia & 56 & 57.1 \\
& Smoking & 28 & 28.6 \\
Complication & Renal abnormality & 3 & 3.1 \\
& Heart abnormality & 17 & 17.3 \\
& Stroke & 1 & 1.0 \\
\hline
\end{tabular}

Table 3 showed the disease characterristics of diabetic patients. The type of DM and duration of disease were asked directly to the patients. As a result, $35.7 \%$ of pati-

Table 3. Diabetes Characteristic of Diabetic Retinopathy Patients in Eye Clinic of Zainoel Abidin Hospital Banda Aceh (categorical data)

\begin{tabular}{llcc}
\hline \multicolumn{1}{c}{ Characteristics } & \multicolumn{1}{c}{ Category } & Frequency $(\mathbf{n = 9 8})$ & Percentage (\%) \\
\hline Type of Diabetes & Type 1 DM & 0 & 0 \\
Mellitus & Type 2 DM & 63 & 64.3 \\
& Unknown & 35 & 35.7 \\
Duration of & Newly diagnosed & 1 & 1.0 \\
Disease & < years & 14 & 14.3 \\
& $5-10$ years & 12 & 12.2 \\
HbA1C Level & $>10$ years & 62 & 63.3 \\
& $<6.5 \%$ & 4 & 6.3 \\
& $6.5-8 \%$ & 19 & 30.2 \\
& $8.1-10 \%$ & 23 & 36.5 \\
& $10.1-12 \%$ & 10 & 15.9 \\
\hline
\end{tabular}

The degree of diabetic retinopathy presented in Table 4 was the type of diabetic retinopathy in one of the eyes, whichever was highest. Most of the patients were present with more severe ocular conditions, such as PDR and DME. Based

ents did not know their DM types. HbA1C levels were obtained from the last laboratory result of patients in the medical record

Table 4. Type of Diabetic Retinopathy Patients in Eye Clinic of Zainoel Abidin Hospital Banda Aceh (categorical data)

\begin{tabular}{llcc}
\hline \multicolumn{1}{c}{ Characteristics } & \multicolumn{1}{c}{ Category } & Frequency (n=98) & Percentage (\%) \\
\hline Type of Diabetic & Mild-moderate NPDR & 13 & 13.3 \\
Retinopathy & Severe-very severe NPDR & 33 & 33.7 \\
& PDR & 52 & 53.0 \\
Diabetic Macular & Present & 51 & 52.0 \\
Edema & Absent & 47 & 48.0 \\
\hline
\end{tabular}


Table 5 showed that most patients were admitted with worse visual acuity. Only less than $10 \%$ of patients had visual acuity of more than 6/12. Ocular complications were commonly found in the patients, indicating the severity of the disease. Neovascular glaucoma was present in $7 \%$ of patients, while tractional retinal detachment was present in $18 \%$ of patients.

Table 5. Other Ocular Findings (categorical data)

\begin{tabular}{llcc}
\hline \multicolumn{1}{c}{ Characteristics } & \multicolumn{1}{c}{ Category } & Frequency (n=98) & Percentage (\%) \\
\hline Right Eye Visual & $\geq 6 / 12$ & 9 & 9.2 \\
Acuity & $>6 / 18-<6 / 12$ & 10 & 10.2 \\
& $>6 / 60-<6 / 18$ & 35 & 35.7 \\
Left Eye Visual & $<6 / 60$ & 44 & 44.9 \\
Acuity & $\geq 6 / 12$ & 8 & 8.2 \\
& $>6 / 18-<6 / 12$ & 8 & 8.2 \\
Other & $>6 / 60-<6 / 18$ & 38 & 38.8 \\
Abnormalities & $<6 / 60$ & 44 & 44.9 \\
& Cataract & 56 & 57.1 \\
Complication & Glaucoma & 3 & 3.1 \\
& Neovascular glaucoma & 2 & 2.0 \\
& Tractional retinal & 7 & 7.1 \\
& detachment (TRD) & 18 & 18.4 \\
\hline
\end{tabular}

\section{DISCUSSION}

It was obtained from this study that most diabetic retinopathy patients (35.7\%) in Eye Clinic of Zainoel Abidin General Hospital Banda Aceh were aged 56 to 65 years. The number of patients was decreased to $13.3 \%$ in the age range $>65$ years. There was no significant difference between female and male sex. This result suited to the previous study conducted by Chatziralli et al. (2010) in Greece which stated that the degree of diabetic retinopathy disease was parallel with age, whereas Xu et al. (2012) in Beijing stated that the highest prevalence of diabetic retinopathy was found in patients aged 50-59 years and the lowest were found in patients aged $\geq 70$ years. Low prevalence of diabetic retinopathy in an older age group is related to increased mortality of DR(Liu et al., 2017).

The complication of diabetes mellitus may affect quality of life and financial conditions of patients and their family. Previous studies demonstrated a correlation between patients with lower socioeconomic background tend to have worse clinical characteristic compared to patients with higher socioeconomic backgrounds. Socioeconomic status is closely related to underlying diabetes treatment, such as quality of health services, family support, disease knowledge, patients' compliance, physical activity, and dietary regiment. This can be observed by educational background, occupation, and monthly salary (Funakoshi et al., 2017; Tao et al., 2016).

This study revealed that most patients (42.9\%) were college graduates. In most epidemiology studies, education was commonly used to determine socioeconomic background. A study conducted by Emoto et al. (2016) stated that people with higher education backgrounds were likely to be more healthy. Patients with lower salaries had the highest prevalence of retinopathy and neuropathy. In this study, most patients worked as housewives with monthly salary were less than 5 million IDR.

Previous studies showed that the incidence and prevalence of hypertension 
in diabetic patients were 1,5 to 2 times greater than non-diabetic patients. Blood pressure is generally normal in the early stage of type 1 diabetes mellitus and increased along with the onset of disease. However, blood pressure is increased in one-third of patients with early-stage of type 2 diabetes mellitus. This study reported 56 respondents (57.1\%) had elevated blood pressure. A study conducted by Faria et al. (2011) in Brazil stated that blood pressure control could reduce superoxide production and/or increase antioxidant defense in the body. Therefore, it would be beneficial for diabetic patients to aim for normal blood pressure in order to delay the progress of diabetic retinopathy and nephropathy. Lipid levels are also one of a significant risk factors for diabetic retinopathy. High lipid levels may lead to endothelial dysfunction due to the low bioavailability of nitric oxide which causes the formation of retinal exudates. This study found 56 respondents (57.1\%) who had a history of high cholesterol levels. This result suited to a study conducted by Cetin et al. (2013) in Turkey which stated that there was a significant correlation between HbA1c and total cholesterol level. However, several past studies demonstrated no correlation between lipid serum levels and diabetic retinopathy. It was suggested that lipid serum levels were involved in the pathogenesis of diabetic macular edema that commonly occurred in late-stage diabetic retinopathy.

Diabetic retinopathy is one of the predictors of cardiovascular disease. It was reported that the risk of stroke and coronary heart disease was higher in diabetic retinopathy patients(Kawasaki et al., 2013). 17 respondents (17.3\%) had a history of heart abnormality and only 1 respondent (1.0\%) had a history of stroke in this study. Renal abnormality was found in 3 respondents (3.1\%). Chronic renal failure and proteinuria are significantly related to diabetic retinopathy. It is caused by chronic hyperglycemia state which leads to a shift in renal and retinal microvascular circulation. This microvascular change results in retinopathy and nephropathy due to inadequate perfusion from narrowed and occluded vascular lumen.

Microvascular complication of diabetes mellitus are firmly linked to severity and duration of hyperglycemia. The disease duration of most patients in this study was $>10$ years $(63.3 \%)$. A study conducted by Yau et al. (2012) in Australia revealed that the prevalence of diabetic retinopathy was parallel to diabetes duration, which presented in21.1\% of patients with $<10$ years diabetes and $76.3 \%$ in patients with $\geq 20$ years diabetes. Proper and comprehensive management of diabetes is essential to prevent complication. Diabetes management relies solely on patients' compliance to medication and lifestyle modification. Therefore, patient education is important to achieve acceptable long-term diabetes treatment result. Past studies showed that patients with decent knowledge of disease had better glycemic control (Al-maskari et al., 2013). Conversely, this study revealed 35 respondents (35.7\%) did not know their diabetes type. As a result, most patients were admitted with advanced disease.

Chronic hyperglycemia is reflected in HbA1c levels. In this study, 23 respondents (36.5\%) had a high level of HbA1c between 8.1-10\%. This result corresponded with a study conducted by Chatziralli et al. (2010) in Greece which found that high level of HbA1c was correlated with severe diabetic retinopathy. $1 \%$ decrease concentration of HbA1c is able to reduce the risk of microvascular complication by 30\% (DeFronzo et al., 2015). 
In 2010 , it was estimated that out of 285 million people with diabetes, one forth had vision-threatening diabetic retinopathy (VTDR). VTDR is defined as severe nonproliferative diabetic retinopathy, proliferative diabetic retinopathy, and diabetic macular edema. Early signs of NPDR are microaneurysm formation, which is ballooning on the capillary wall. Later, when retina capillary hypoperfusion becomes more severe, neovascularization appears as a sign of PDR (Kollias and Ulbig, 2010). In this study, each right and left eye of subject were examined and grouped based on the more severe degree of diabetic retinopathy. Most respondents (50.0\%) were admitted with PDR in one of the eyes. Moreover, diabetic macular edema was also found in 51 respondents (52.0\%).

Previous studies demonstrated that in 2010, 3.63 million people worldwide experienced moderate and severe loss of vision due to diabetic retinopathy. Moderate and severe loss of vision is defined as visual acuity of $3 / 60$ to $6 / 18$ on the Snellen chart. On the other hand, it is estimated that 850 thousand people experienced blindness with visual acuity less than $3 / 60$. This study showed that right or left eye visual acuity in 44 respondents (44.9\%) was less than 6/60. Diabetes is also one of the risk factors for early-stage cataract, whereas cataract is a significant factor that causes loss of vision and blindness in diabetic patients. Cataract was present in 56 respondent (57.1\%) from this study. Past studies also reported diabetes as one of the risk factors for glaucoma, especially in patients with proliferative diabetic retinopathy. Neovascular glaucoma was reported in 7 respondents (7.1\%) in this study. Worse visual acuity followed by ocular complication in some patients is associated with knowledge, attitude, and behavior patients in relation to their disease. Up to $50-73 \%$ of visual loss or blindness cases caused by diabetic retinopathy can be prevented by early detection and early management of risk factors. Screening for diabetic retinopathy is best done early in the course of diabetes mellitus. Patients need to understand the risk of diabetes complications, such as diabetic retinopathy, which does not exhibit any symptoms early in the course of the disease (Petrella et al., 2012).

To conclude, this study found that most patients came with advance disease (PDR) with visual acuity of less than 6/60. Information and knowledge regarding diabetic retinopathy is essential for all diabetes mellitus patients. They must be informed that in the early stage, the disease is asymptomatic, and that ophthalmologists recommend all diabetic patients to undergo periodic examination for early detection. This study showed that diabetic retinopathy patients had been diagnosed as diabetic for more than 10 years, followed by worse visual acuity and abnormal HbAic levels. The result indicates poor behavior and knowledge of patients in regards to their disease. Further studies are required to examine the attitude, behavior, and knowledge of diabetic patients in Aceh concerning diabetic retinopathy or other ocular diabetic complications to prevent irreversible visual damage and improve patients' quality of life.

\section{AUTHOR CONTRIBUTION}

The authors actively participated in this study. Lia Meuthia Zaini and Sri Marlinda collected the data from medical records, interviewed patients to obtain additional information that is necessary for this study. All writers contributed to the scriptwriting and the revision of the manuscripts. 
Zaini et al./ Clinical Characteristics of Diabetic Retinopathy Patients

\section{CONFLICT OF INTEREST}

The authors declare that the study was conducted without conflict of interest for the publication of this article.

\section{FUNDING AND SPONSORSHIP}

The authors do not receive any financial support or sponsorship for this article journal.

\section{ACKNOWLEDGEMENT}

The authors acknowledge the support from Zainoel Abidin General Hospital, Banda Aceh for permitting to conduct this study.

\section{REFERENCE}

Al-maskari F, El-Sadig M, Al-Kaabi J, Afandi B, Nagelkerke N, Yeatts K (2013). Knowledge, attitude and practices of diabetic patients in the United Arab Emirates. PLoS ONE. 8(1): 1-8. https://doi.org/10.1371/journal.pone. 0052857 .

Cheung N, Wong TY (2008). Diabetic retinopathy and systemic vascular complications. Progress in Retinal and Eye Research. 27: 161-176. https://doi.org/10.1016/j.preteyeres.2007.12. 001.

DeFronzo R, Ferrannini E, Groop L, Henry R, Herman W, Holst J, Hu F, et al. (2015). Type 2 diabetes mellitus. Nat Rev Dis Primers. 1: 15019. https://doi.org/10.1038/nrdp.2015.19.

Ding J, Wong TY (2012). Current epidemiology of diabetic retinopathy and diabetic macular edema. Curr Diab Rep. 12: 346-354. https://doi.org/10.1007/s11892-012-0283-6.

Duh E J, Sun JK, Stitt AW (2017). Diabetic retinopathy: current understanding, mechanisms, and treatment strategies. JCI Insight. 2(14):1-13. https://doi.org/10.1172/jci.insight.93751.

Funakoshi M, Azami Y, Matsumoto H,
Ikota A, Ito K, Okimoto H, Shimizu N, et al. (2017). Socioeconomic status and type 2 diabetes complications among young adult patients in Japan. PLoS ONE. 12(4): 1-14. https://doi.org/10.1371/journal.pone.0176087.

Kawasaki R, Tanaka S, Tanaka S, Abe S, Sone H, Yokote K, Ishibashi S, et al. (2013). Risk of cardiovascular diseases is increased even with mild diabetic retinopathy the Japan Diabetes Complications Study. Ophthalmology. 120(3): 574-582. https://doi.org/10.1016/j.ophtha.2012.08.029.

Khandekar R, Harby SA, Harthy HA, Lawatti JA (2010). Knowledge, attitude and practice regarding eye complications and care among Omani persons with diabetes - A cross sectional study. Oman J Ophthalmol. 3(2): 6065. https://doi.org/10.4103/0974-62ox.64228.

Kollias AN, Ulbig MW (2010). Diabetic retinopathy early diagnosis and treatment. Dtsch Arztebl Int. 107(5): 7584. https://doi.org/10.3238/arztebl.2010.0075.

Lee R, Wong TY, Sabanayagam C(2015). Epidemiology of diabetic retinopathy, diabetic macular edema and related vision loss. Eye and Vision. 2(17): 125. https://doi.org/10.1186/s40662015-0026-2.

Liu Y, Yang J, Tao L, Lv H, Jiang X, Zhang $M$, Li X (2017). Risk factors of diabetic retinopathy and sight-threatening diabetic retinopathy: a cross-sectional study of 13473 patients with type 2 diabetes mellitus in mainland China. BMJ Open. 7: 1-11. https://doi.org/10.1136/bmjopen-2017-016280.

Nentwich MM, Ulbig MW (2015). Diabetic retinopathy - ocular complications of diabetes mellitus.World Journal of Diabetes. 6(3): 489-499. https://doi.- 
Zaini et al./ Clinical Characteristics of Diabetic Retinopathy Patients

org/10.4239/wjd.v6.i3.489.

Petrella RJ, Blouin J, Davies B, Barbeau M (2012). Prevalence, demographics, and treatment characteristics of visual impairment due to diabetic macular edema in a representative Canadian cohort. J Ophthalmol. 159167. https://dx.doi.org/10.1155\%2F2012\%2F159 167.

Sayin N, Kara N, Pekel G (2015). Ocular complications of diabetes mellitus. World J Diabetes. 6(1): 92-108. https://doi.org/10.4239/wjd.v6.i1.92.

Tajunisah I, Wong PS, Tan LT, Rokiah P, Reddy SC (2011). Awareness of eye complications and prevalence of retinopathy in the first visit to eye clinic among type 2 diabetic patients. Int $\mathrm{J}$ Ophthalmol. 4(5): 519-524. https://doi.org/10.3980/j.issn.2222-3959.20-
11.05.12.

Tao X, Li J, Zhu X, Zhao B, Sun J, Ji L, Hu $\mathrm{D}$, et al. (2016). Association between socioeconomic status and metabolic control and diabetes complications: a cross-sectional nationwide study in Chinese adults with type 2 diabetes mellitus.Cardiovas Diabetol. 15(61): 1-10. https://doi.org/10.1186/s12933-016-0376-7.

Wat N, Wong R LM, Wong IYH (2016). Associations between diabetic retinopathy and systemic risk factors. Hong Kong Med J. 22(6): 589-599. https://doi.org/10.12809/hkmj164869.

Zheng Y, He M, Congdon N (2012). The worldwide epidemic of diabetic retinopathy. Indian Journal of Ophthalmology. 6o(5):428-431. https://doi.org/10.4103/0301-4738.100542. 